\title{
Collaborative Spectrum Sensing: Optimising the number of collaborating users
}

\author{
Kamran Arshad and Klaus Moessner \\ Centre for Communication Systems Research \\ University of Surrey, GU2 7XH, UK \\ Email:k.arshad@surrey.ac.uk
}

\begin{abstract}
In the IEEE 802.22 standard, the spectrum sensing mechanism is identified as a key functionality of a cognitive radio. Due to the channel uncertainty, a single cognitive user, in most cases, can not make a reliable decision and hence collaboration or cooperation of and among multiple users is required. However, when large number of cognitive users are collaborating with each other, the bandwidth requirements for sending their result to the fusion centre tends to be very large. In this paper, a metric for spectrum efficiency is defined and used for the optimisation of collaborative spectrum sensing. An optimisation algorithm is presented to calculate the optimal number of collaborating cognitive users with the aim to maximise overall spectrum efficiency by satisfying certain constraints in terms of global probability of detection and probability of false alarm. Numerical results show that for maximum spectrum efficiency collaboration of only a subset of the available cognitive users is required.
\end{abstract}

\section{INTRODUCTION}

Cognitive radio (CR) is an emerging technology to enhance spectrum utilisation without affecting the transmission of licensed system [1]. Spectrum sensing is a key functionality of a CR and it must be performed before the CR starts its transmission in the licensed band in order to limit the interference to the primary user (PU) [2]. However, due to the channel effects, spectrum sensing performed by a single user is not reliable and hence collaborative spectrum sensing has been proposed [3]-[6].

Collaborative spectrum sensing has two main phases: sensing and reporting. In the sensing phase, each individual CR makes an observation using local spectrum sensing algorithm, while in the reporting phase, each CR sends its observation (or decision) to the fusion centre. The energy detection is optimal for detecting any unknown deterministic signal and hence used in this work as an underlaying local spectrum sensing scheme [7]. Fusion centre combine local observations (soft decision combining) or decisions (hard decision combining) and make the final decision to indicate the presence or absence of the PU. This final decision is then broadcast to all collaborating cognitive users. It has been argued that hard decision combining is a better choice than soft decision combining due to its significantly lower communication overhead [5].

In order to evaluate the performance of spectrum sensing, two probabilities are of great interest: probability of detection and the probability of false alarm. Probability of detection $P_{d}$ determines the level of interference-protection provided to the PU while probability of false alarm $P_{f a}$ indicates percentage of white spaces falsely declared as occupied [5]. In the context of opportunistic spectrum access, $P_{d}$ must be higher than some pre-defined threshold while $P_{f a}$ should be lower than some desired criteria or as minimum as possible for better spectrum utilisation.

Collaborative spectrum sensing, even in hard decision combining, needs a reporting channel for each user to send its decision to the fusion centre; reporting channels are usually bandwidth limited [8]. In the literature, spectrum efficiency was improved by mainly utilising censoring sensors in which each cognitive radio censored its observation before sending it to the fusion centre [9], [10]. In censoring sensing methods, only users having enough information are allowed to send their decision to the fusion centre. Censoring method using double threshold was also proposed to reduce communication traffic overhead in [9], [11]. An optimisation algorithm to satisfy total error bound is described recently in [12] for an AWGN channel. Similarly, optimisation of cooperative spectrum sensing in terms of number of users for the case of AND and OR fusion rule in AWGN channel was described in [13].

In this paper, optimal number of collaborative cognitive users are calculated to maximise spectrum efficiency in Rayleigh fading channel. A new metric that expresses spectrum efficiency is defined to evaluate the performance of collaborative spectrum sensing overhead. Spectrum efficiency can be increased by keeping probability of false alarm low and by minimising the number of collaborating cognitive users to a minimum. In cognitive radio network, collaboration of a large number of users is impractical because in one time slot only one cognitive radio can send its decision to the fusion centre in order to separate decisions easily at the fusion centre. An optimisation algorithm is presented, to calculate the optimum number of collaborating users to maximise spectrum efficiency by satisfying some spectrum sensing constraints in terms of probability of detection and probability of false alarm. In particular, first spectrum efficiency is defined and then an expression for the spectrum efficiency is derived for the scenario considered in this paper. Proposed algorithm then calculates optimal number of users to maximise spectrum efficiency by satisfying bounds on the global probability of detection and the probability of false alarm.

The rest of the paper is structured as follows. In section II the system model and collaborative spectrum sensing is 
briefly introduced. A new metric of spectrum efficiency is defined and a mathematical expression for spectrum efficiency is derived in section III. An optimisation algorithm to calculate optimal number of collaborating users for given constraints is described in section III. The simulation results are shown in section IV. Finally, section V concludes this paper.

\section{SySTEM MODEL}

We consider a cognitive radio network, with $M$ collaborative cognitive users and a fusion centre, to sense a portion of the spectrum in order to detect the PU, as shown in Fig. 1. We assume that PU is far away from the group of $M$ cognitive users and hence same mean SNR can be assume for all users. It is also assumed that all cognitive radios have independent and indentically distributed (i.i.d.) observations. Each CR makes its own decision and then selected number of cognitive radios transmit their decision to the fusion centre via reporting channel. Final decision will be made at the fusion centre and then the information about available white spaces will be broadcast to all $M$ cognitive radios operating in a particular area.

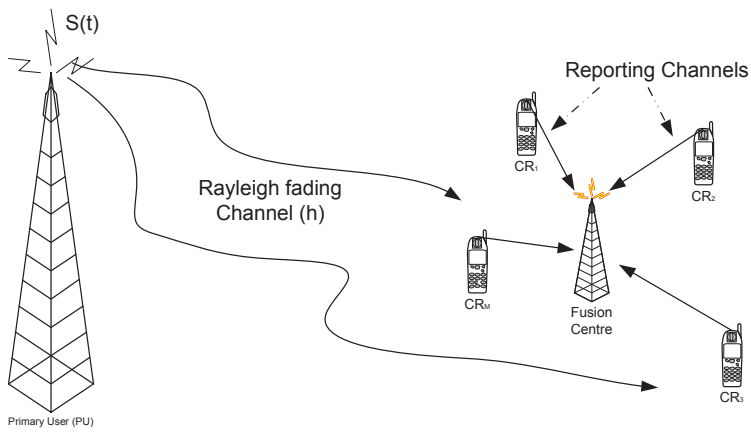

Fig. 1. Cognitive radio network sensing primary user signal

\section{A. Spectrum Sensing}

Spectrum sensing at a local CR node is considered as a binary hypothesis testing problem with two possible hypotheses $\mathcal{H}_{0}$ and $\mathcal{H}_{1}$, defined as [14],

$$
x(t)= \begin{cases}n(t), & ; \mathcal{H}_{0} \\ h s(t)+n(t), & ; \mathcal{H}_{1}\end{cases}
$$

where $s(t)$ is the PU signal, $n(t)$ is assumed to be Gaussian i.i.d. random process with zero mean and variance $\sigma_{n}^{2}(t)$ and $h$ is the amplitude gain of the channel. Further, it is assumed that $s(t)$ and $n(t)$ are independent of each other.

PU signal detection is performed using an energy detector at ith cognitive radio and compute decision statistics $Y_{i}$ which corresponds to energy collected in observation time $T$ and bandwidth $W$, and described in [15]:

$$
Y_{i}= \begin{cases}\chi_{2 m}^{2}, & ; \mathcal{H}_{0} \\ \chi_{2 m}^{2}\left(2 m \overline{\gamma_{i}}\right), & ; \mathcal{H}_{1}\end{cases}
$$

where $\chi_{2 m}^{2}$ is a chi-square distribution with $2 m$ degrees of freedom, $\chi_{2 m}^{2}\left(2 m \bar{\gamma}_{i}\right)$ is non-central chi-square distribution with $2 m$ degrees of freedom and a non-centrality parameter of $2 m \bar{\gamma}_{i}, m=T W$ is the time bandwidth product and $\bar{\gamma}_{i}$ is the signal to noise ratio (SNR) at the ith $\mathrm{CR}$ terminal. Decision statistics $Y_{i}$ are compared with a pre-defined threshold $\lambda_{i}$ that is selected to satisfy false alarm rate specifications of the detector to make a decision $\mathcal{H}_{0}$ or $\mathcal{H}_{1}$ as defined in (1).

$P_{d}^{i}$ and $P_{f a}^{i}$ are defined as the probabilities that an $i t h$ CR detects a primary signal under hypothesis $\mathcal{H}_{1}$ and $\mathcal{H}_{0}$, respectively. For ith user in AWGN channel, probability of detection $P_{d}^{i}$ and probability of false alarm $P_{f a}^{i}$ is given by [14]:

$$
\begin{aligned}
P_{f a}^{i} & =\operatorname{Prob}\left\{Y_{i}>\lambda_{i} \mid \mathcal{H}_{0}\right\} \\
& =\frac{\Gamma\left(m, \lambda_{i} / 2\right)}{\Gamma(m)} \\
P_{d}^{i} & =\operatorname{Prob}\left\{Y_{i}>\lambda_{i} \mid \mathcal{H}_{1}\right\} \\
& =Q_{m}\left(\sqrt{2 m \bar{\gamma}_{i}}, \sqrt{\lambda_{i}}\right)
\end{aligned}
$$

where $\lambda_{i}$ denotes the threshold at $i t h$ user, $\Gamma(a)$ is the gamma function, $\Gamma(a, x)$ is incomplete gamma function and $Q_{m}(a, b)$ is the generalised Marcum Q-function (for definition see [16]).

When the channel gain $h$ is varying due to the fading or shadowing, then $P_{d}^{i}$ is conditional probability dependent on instantaneous SNR $\gamma_{i}$. In this case, average probability of detection (by abuse of notation still denoted by $P_{d}^{i}$ ) can be obtained by averaging $P_{d}$ over fading statistics $f_{\gamma}(x)$ [5]:

$$
P_{d}^{i}=\int_{\gamma} Q_{m}(\sqrt{2 m \bar{\gamma}}, \sqrt{\lambda}) f_{\gamma}(x) d x
$$

and for a rayleigh fading channel, $P_{d}^{i}$ is given by [14],

$$
\begin{aligned}
& P_{d}^{i}=e^{-\frac{\lambda_{i}}{2}} \sum_{n=0}^{m-2} \frac{1}{n !}\left(\frac{\lambda_{i}}{2}\right)^{m} \\
& +\left(\frac{1+\bar{\gamma}_{i}}{\bar{\gamma}_{i}}\right)^{m-1}\left[e^{-\frac{\lambda_{i}}{2\left(1+\bar{\gamma}_{i}\right)}}-e^{-\frac{\lambda_{i}}{2}} \sum_{n=0}^{m-2} \frac{1}{n !} \frac{\lambda_{i} \bar{\gamma}_{i}}{2\left(1+\bar{\gamma}_{i}\right)}\right]
\end{aligned}
$$

It has been shown many times in the literature that spectrum sensing performance can be greatly improved with the collaborative or cooperative spectrum sensing in which a number of cognitive radios share their sensing information with each other [3]-[6]. In this paper, OR fusion rule is used, in which when one out of $M$ cognitive radios detect the PU, fusion centre declares a PU is present [5]. The global probability of detection and false alarm is then given by,

$$
\begin{aligned}
Q_{d} & =1-\prod_{i=1}^{M}\left(1-P_{d}^{i}\right) \\
Q_{f a} & =1-\prod_{i=1}^{M}\left(1-P_{f a}^{i}\right)
\end{aligned}
$$

For the system model shown in Fig. 1, it is clear that all cognitive radios experience similar path loss and hence $\bar{\gamma}_{i}=$ $\bar{\gamma}, \lambda_{i}=\lambda, \forall i \in\{1,2, \ldots, M\}$. 


\section{Optimisation of Collaborating Cognitive RADIOS}

\section{A. Spectrum Efficiency}

In collaborative spectrum sensing, efficiency of spectrum utilisation depends on two factors: Global probability of false alarm $Q_{f a}$ and the number of collaborating cognitive users $M_{\text {sel }}$ participating in collaborative spectrum sensing, where $1 \leq M_{\text {sel }} \leq M$. Based on these two factors, spectrum efficiency can be defined as,

$$
\eta=\frac{\alpha\left(1-\frac{M_{\mathrm{sel}}}{M}\right)+(1-\alpha)\left(1-Q_{f a}\right)}{2}
$$

where $\alpha$ is a scaling factor.

By defining local probability of detection as $P_{d}^{i} \triangleq G_{m}(\lambda)$, probability of false alarm for $i$ th user can be represented as,

$$
P_{f a}^{i}=\frac{\Gamma\left(m, G_{m}^{-1}\left(P_{d}^{i}\right) / 2\right)}{\Gamma(m)}
$$

For a desired value of global probability of detection $Q_{d}=$ $Q_{d 0}$ and $M_{\text {sel }}$ users, (6) can be re-written as,

$$
P_{d}^{i}=1-\sqrt[M_{\mathrm{sel}}]{1-Q_{d 0}}
$$

and threshold $\lambda$ is,

$$
\lambda=G_{m}^{-1}\left[1-\sqrt[M_{\mathrm{sel}}]{1-Q_{d 0}}\right]
$$

From (7), (9) and (10),

$$
Q_{f a}=1-\left\{1-\frac{\Gamma\left(m, \frac{1}{2} G_{m}^{-1}\left(1-M_{\mathrm{sel}} / 1-Q_{d 0}\right)\right.}{\Gamma(m)}\right\}^{M_{\mathrm{sel}}}
$$

Substitute (12) in (8), spectrum efficiency $\eta$ is given by,

$$
\begin{aligned}
\eta= & \frac{\alpha}{2}\left\{1-\frac{M_{\mathrm{sel}}}{M}\right\}+\frac{(1-\alpha)}{2} \\
& \left\{1-\frac{\Gamma\left(m, \frac{1}{2} G_{m}^{-1}\left(1-M_{\mathrm{sel}} \sqrt{1-Q_{d 0}}\right)\right)}{\Gamma(m)}\right\}^{M_{\mathrm{sel}}}
\end{aligned}
$$

The problem is to find out the optimum number of collaborating cognitive users i.e. $M_{\text {sel }}$, which maximise spectrum efficiency i.e. (13) under given constraints. Optimisation constraint in this problem is defined as the global probability of false alarm i.e. optimum number of cognitive radios must satisfy $Q_{f a}\left(M_{\text {sel }}\right) \leq Q_{f 0}$ where $Q_{f 0}$ is some pre-defined constraint on global probability of false alarm.

Hence optimisation problem is defined as,

$$
\begin{array}{cc} 
& \max _{M_{\text {sel }}} \eta\left(M_{\text {sel }}\right) \\
\text { s.t. } & 1 \leq M_{\text {sel }} \leq M \quad \text { and } \quad Q_{f a}\left(M_{\text {sel }}\right) \leq Q_{f 0}
\end{array}
$$

\section{B. Optimum number of cognitive users}

It is normally difficult to find a close-form solution of (14) especially for the case of Rayleigh fading channel. We propose an optimisation algorithm to calculate minimum number of users $M_{\text {sel }}$ which maximise spectrum efficiency and satisfies spectrum sensing constraint as defined in (14).

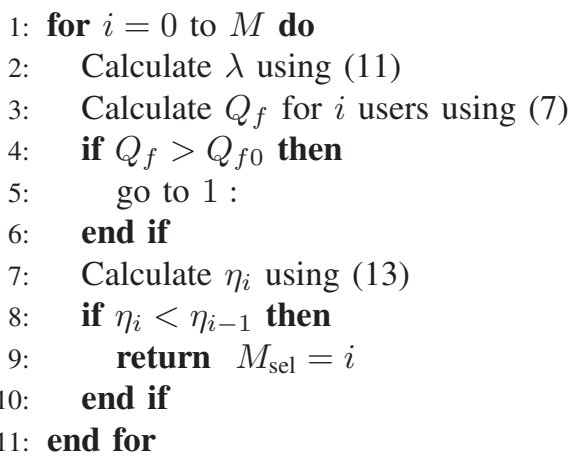

\section{Numerical Results AND Discussion}

In this section the proposed collaboration scheme and algorithm is evaluated numerically. We assume that there is a large number of users in a cognitive radio network and all collaborating users have same mean SNR, as discussed in section II. For all results, level of protection to the licensed system in terms of global probability of detection is assumed as $Q_{d 0}=0.99$ and threshold for global probability of false alarm is assumed to be $Q_{f 0}=10^{-2}$.

Fig. 2 plots spectrum efficiency versus number of collaborating cognitive users when the mean SNR for the cognitive radio network is $\gamma_{d B}=0$. As seen from Fig. 2 that maximum spectrum efficiency is obtained by collaboration of 30 cognitive users and by increasing number of users, spectrum efficiency drops (as more reporting channel bandwidth is required to report individual observations to the fusion centre). Although, 30 users can provide maximum spectrum efficiency but they can not gaurantee spectrum sensing constraints i.e. $Q_{f a} \leq 10^{-2}$. As seen from Fig. 2 that the number of optimal users calculated by the proposed algorithm described in III are 148. Hence, in AWGN channel with $\gamma_{d B}=0$, collaboration of all 500 radios is not required and only 148 users are enough to maximise spectrum efficiency and to maintain desired constraints on $Q_{f a}$ and $Q_{d}$.

Spectrum efficiency gain is defined as the gain in terms of bandwidth saving by employing $M_{\text {sel }}$ out of $M$ collaborating users. For the case of AWGN channel, $\gamma_{d B}=0, Q_{d 0}=0.99$ and $Q_{f 0}=10^{-2}$ spectrum efficiency gain is reported as 17.44\%. Similarly, Fig. 3 shows spectrum efficiency versus number of collaborating cognitive radios in Rayleigh fading channel, and it is shown that for desired performance number of collaborating radios to maximise spectrum efficiency are 269 , which gives spectrum efficiency gain of $11.38 \%$.

Another important metric to characterise collaborative spectrum sensing performance is the minimum detectable SNR that can be detected by a cognitive radio network. This metric is 


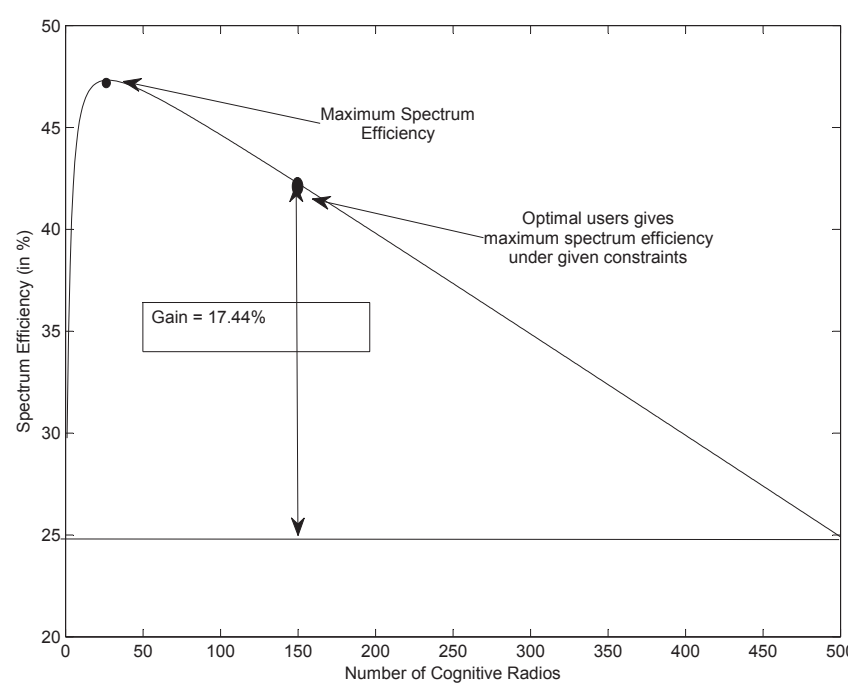

Fig. 2. Spectrum Efficiency versus number of collaborating cognitive use in AWGN channel

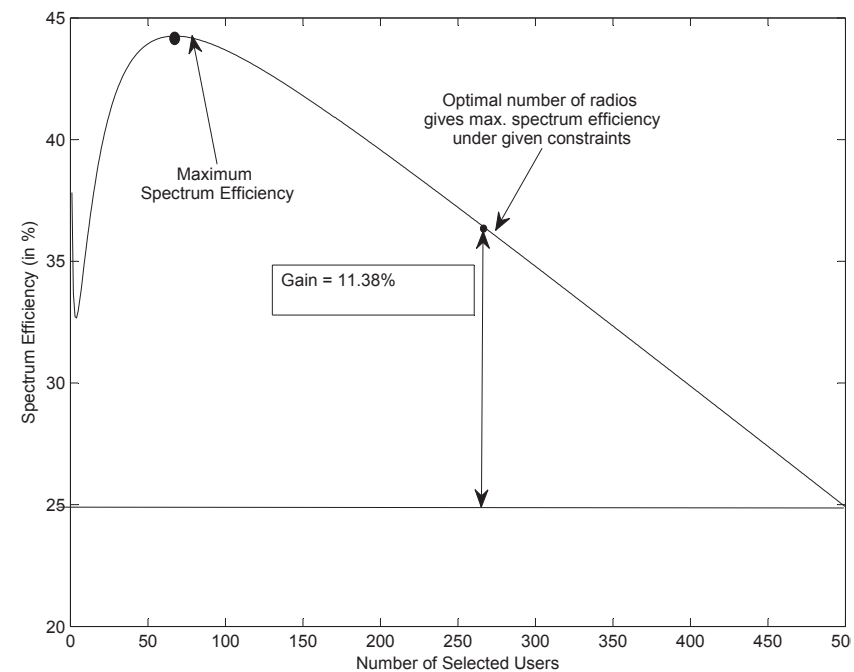

Fig. 3. Spectrum Efficiency versus number of collaborating cognitive use in Rayleigh fading channel

defined as the minimum mean SNR that can be detected by a cognitive radio with reliability for given $P_{f a}$ and $P_{d}$, primary user signal, channel conditions and observation time. Fig. 4 shows maximum achievable spectrum efficiency by employing optimal number of cognitive users versus minimum detectable SNR in an AWGN and a Rayleigh fading channel. In order to detect a licensed signal of $\gamma_{d B}=4$ in AWGN channel, maximum spectral efficiency is $49.66 \%$ while in Rayleigh fading channel maximum spectrum efficiency is $36.25 \%$. This is due to the fact that in Rayleigh fading channel, in order to detect the same mean SNR more number of collaborative cognitive radios are needed to guarantee same spectrum sensing performance in terms of $Q_{d}$ and $Q_{f a}$. Similarly, Fig. 5 plots number of optimal collaborative cognitive radios versus minimum detectable SNR and it is clear from the Fig. 5 that more number of collaborative partners are needed to overcome channel effects.

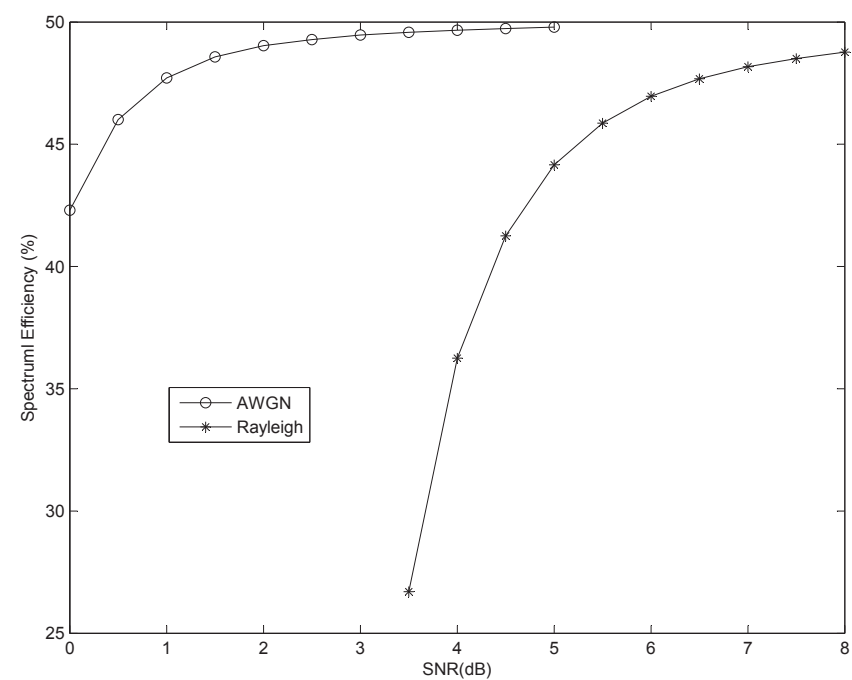

Fig. 4. Spectrum Efficiency versus minimum detectable SNR

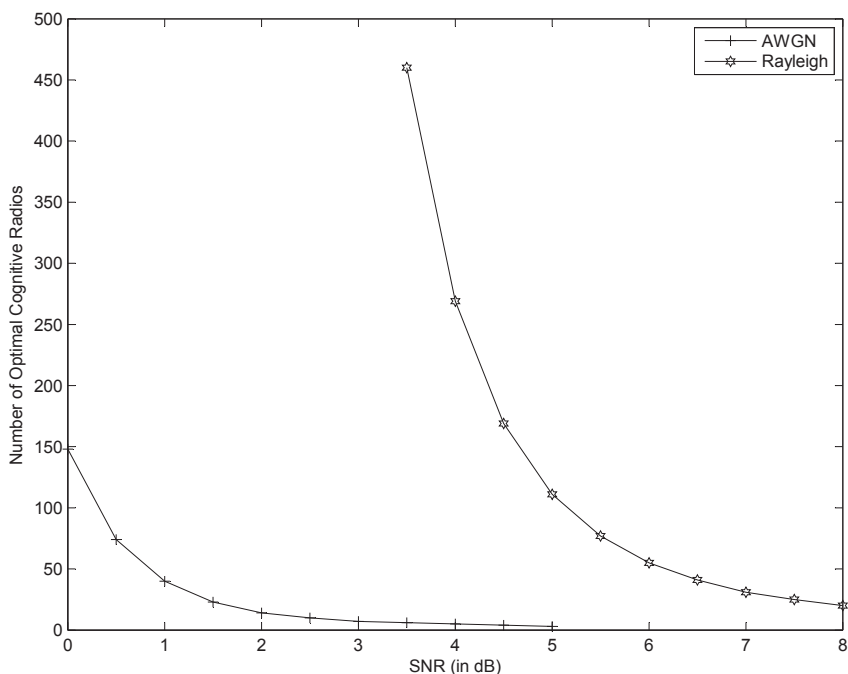

Fig. 5. Number of Collaborative cognitive users versus minimum detectable SNR

\section{CONCLUSIONS}

Optimisation of collaborative spectrum sensing for cognitive radio network to maximise spectrum efficiency has been studied. A new metric of spectrum efficiency for collaborative spectrum sensing is defined and an algorithm is presented to find optimal number of users in a Rayleigh fading channel to maximise spectrum efficiency with the constraint of detection and false alarm probabilities. It is shown here that spectrum efficiency is dependent on the number of collaborating radios and optimum value of spectrum efficiency can be obtained if certain number of cognitive radios collaborate with each others. Moreover, spectrum efficiency gains are quantised 
in this paper for the case of AWGN and Rayleigh fading channels.

\section{ACKNOWLEDGMENT}

This work was performed in the project E3 which has received research funding from the EU FP7 framework. The contributions of colleagues from the E3 consortium are hereby acknowledged.

\section{REFERENCES}

[1] I. Akyildiz, W.-Y. Lee, M. Vuran, and S. Mohanty, "A survey on spectrum management in cognitive radio networks," Communications Magazine, IEEE, vol. 46, no. 4, pp. 40-48, April 2008.

[2] D. Datla, R. Rajbanshi, A. Wyglinski, and G. Minden, "Parametric adaptive spectrum sensing framework for dynamic spectrum access networks," in 2nd IEEE International Symposium on New Frontiers in Dynamic Spectrum Access Networks, DySPAN'07, April 2007, pp. 482485.

[3] D. Cabric, A. Tkachenko, and R. Brodersen, "Spectrum sensing measurements of pilot, energy, and collaborative detection," in IEEE Military Communications Conference, MILCOM'06, Oct. 2006, pp. 1-7.

[4] K. Arshad and K. Moessner, "Collaborative spectrum sensing for cognitive radio," in 2009 Joint Workshop on Cognitive Wireless Networks and Systems, 2009.

[5] A. Ghasemi and E. S. Sousa, "Opportunistic spectrum access in fading channels through collaborative sensing," Journal of Communications, vol. 2, no. 2, pp. 71-81, March 2007.

[6] X. Zheng, L. Cui, J. Chen, Q. Wu, and J. Wang, "Cooperative spectrum sensing in cognitive radio systems," in Congress on Image and Signal Processing, CISP'08, vol. 5, May 2008, pp. 262-266.
[7] A. Sahai, N. Hoven, and R. Tandra, "Some fundamental limits on cognitive radio," in Forty-second Allerton Conference on Communication, Control, and Computing, 2004.

[8] D. Cabric, S. Mishra, and R. Brodersen, "Implementation issues in spectrum sensing for cognitive radios," in Thirty-Eighth Asilomar Conference on Signals, Systems and Computers, vol. 1, Nov. 2004, pp. 772-776.

[9] C. Sun, W. Zhang, and K. Ben, "Cluster-based cooperative spectrum sensing in cognitive radio systems," in IEEE International Conference on Communications, ICC'07, June 2007, pp. 2511-2515.

[10] R. Jiang and B. Chen, "Fusion of censored decisions in wireless sensor networks," Wireless Communications, IEEE Transactions on, vol. 4, no. 6, pp. 2668-2673, Nov. 2005.

[11] J. Zhu, Z. Xu, F. Wang, B. Huang, and B. Zhang, "Double threshold energy detection of cooperative spectrum sensing in cognitive radio," in Cognitive Radio Oriented Wireless Networks and Communications, 2008. CrownCom 2008. 3rd International Conference on, May 2008, pp. $1-5$.

[12] W. Zhang, R. Mallik, and K. Ben Letaief, "Cooperative spectrum sensing optimization in cognitive radio networks," in IEEE International Conference on Communications, ICC'08, May 2008, pp. 3411-3415.

[13] E. Peh and Y.-C. Liang, "Optimization for cooperative sensing in cognitive radio networks," in IEEE Wireless Communications and Networking Conference, WCNC'07, March 2007, pp. 27-32.

[14] F. F. Digham, M.-S. Alouini, and M. K. Simon, "On the energy detection of unknown signals over fading channels," IEEE Transactions on Communications, vol. 55, no. 1, pp. 21-24, Jan. 2007.

[15] H. Urkowitz, "Energy detection of unknown deterministic signals," IEEE Proceedings, vol. 55, no. 4, pp. 523-531, April 1967.

[16] I. S. GRADSHTEYN and I. RYZHIK, Table of integrals, series and products. New-York: Academic Press, 1980, incorporating the 4th ed. prepared by Yu. V. Geronimus and M. Yu. Tseytlin. 